\title{
Prevalence of primary basilar invagination among asymptomatic Sri Lankan adults - computed tomographic study
}

Kodiara $\mathrm{I}^{1}$, Gamage $\mathrm{D}^{2}$, Ilayperuma $\mathrm{I}^{1}$

${ }^{\prime}$ Department of Anatomy, Faculty of Medicine, University of Ruhuna, Galle, Sri Lanka. ${ }^{2}$ Teaching Hospital, Rathnapura, Sri Lanka.

\begin{abstract}
Objective: To evaluated the prevalence and some associated factors of basilar invagination (BI) in a group of Sri Lankan adults.
\end{abstract}

\section{Material and methods: Brain} computed tomographic images (CT) of adults admitted to Teaching Hospital Rathnapura, Sri Lanka were studied, excluding subjects with craniofacial syndromes, cerebral or spinal pathologies. The distance between Chamberlain's line (CL) and odontoid process (OP) measured in sagittal $\mathrm{CT}$ images (bone window). $\mathrm{BI}$ is defined when the OP is $2 \mathrm{~mm}$ above CL.

Results: The study group ( $\mathrm{n}=135$; male $=67$; female $=68$ ) was in 20-97 years age range (mean- $56 \pm 18$ years). BI was present in $11.8 \%(n=16)$, of which the mean position of OP was $2.33 \pm 1.06 \mathrm{~cm}$ above $\mathrm{CL}$ (range=0.6-4.7 $\mathrm{mm})$. In $13.4 \%$, OP was at the same level as CL. When BI is absent, the mean position of OP was $2.1 \pm 1.9 \mathrm{~cm}$ below CL. The OP was below CL in $86.6 \%(n=58)$ of males and $75 \%(n=51)$ of females demonstrating no significant $(\mathrm{X} 2=2.904 ; \mathrm{p}=0.088)$ gender variation in BI. Internal cranial height $(\mathrm{OR}=0.823 ; 95 \% \mathrm{CI}=0.678-0.990$; $\mathrm{p}=0.044$ ) was negatively associated with the incidence of BI. However, age $(\mathrm{OR}=1.015 ; 95 \% \mathrm{CI}=0.984-1.047$; $\mathrm{p}=0.341)$, gender $(\mathrm{OR}=0.707$; $95 \% \mathrm{CI}=0.226-2.218 ; \mathrm{p}=0.553)$, posterior fossa height $(\mathrm{OR}=0.912$; 95\% $\mathrm{CI}=0.808-1.030 ; \mathrm{p}=0.136)$, or Klaus' height index (OR=1.099; 95\% $\mathrm{CI}=0.874-1.383 ; \mathrm{p}=0.419$ ) had no significant influence on BI.

Conclusion: Current study, for the first time, documents the incidence of BI among asymptomatic Sri Lankans. Since ethnic variation in the incidence of $\mathrm{BI}$ is reported, such populationspecific data would be invaluable in diagnosing the asymptomatic BI cohort, which in turn, will facilitate to prevent permanent disability and lifethreatening emergencies.

Keywords: basilar invagination, prevalence, asymptomatic patients, computed tomography

\section{Introduction}

The anatomy of craniocervical junction attracts great attention considering the vital neural structures clustered in this sophisticated region, in which the relationship of the odontoid process of the second cervical vertebra to the 
Kodiara I, Gamage D, Ilayperuma I - Prevalence of primary basilar invagination among asymptomatic Sri Lankan adults - computed tomographic study

upper cervical spinal cord, cerebellar tonsils and the brain stem are critical $(1,2,4,5)$. Under normal conditions, the odontoid process does not compress or impinge on any of the adjacent neural structures. However, the possibility of the brain stem or spinal cord impingement by the odontoid process can be increased by many factors, such as developmental abnormalities, variations in normal positional or altered position due to a pathological process (1,2,4-6). Basilar invagination (BI) or the superior migration of the odontoid process and Chiari malformation are the most frequent of the craniocervical region abnormalities causing neural compression, commonly resulted from a developmental defect of the occiput (7).

The majority of patients with BI are asymptomatic and develop symptoms following a trivial injury or infection in the head and neck region $(2,3,6)$. The symptomatic minority (BI patients) require substantial medical care to prevent adverse squeal of neural compression. Unless treated, the majority of symptomatic BI patients may end up in permanent disability or even succumbed to death $(2,4,8-10)$. Importantly, even a certain proportion of asymptomatic patients have shown the potential of developing permanent disability following trivial trauma.

Therefore, diagnosing $\mathrm{BI}$ is essential before the complications manifest $(2,6)$

Since the clinical diagnosis of BI is challenging due to the non-specific presentation in the majority; investigations, particularly the imaging investigations play a pivotal role. In the radiological diagnosis of $\mathrm{BI}$, violation of the Chamberlain's line by the tip of the odontoid process is considered. Many authors used different thresholds to define BI: the tip of odontoid process $2,3,5$ and $6 \mathrm{~mm}$ above the CL $(2,5,8,9)$. The lateral view of the $\mathrm{X}$-ray cervical spine has been used in diagnosis before the dawn of the era of computed tomography (CT) $(6,8,11)$. The CT cervical spine with sagittal reconstruction is more sensitive and specific than X-ray in diagnosing BI due to several reasons. Firstly, the measurement accuracy of the electronic measurement calliper system is high. Secondly, with multi-planner image reconstruction ability, the $\mathrm{CT}$ offers a high resolution of the imaged structures $(1,10)$. Compared to CT, magnetic resonant imaging (MRI) offers similar or higher sensitivity in detecting $\mathrm{BI}$; however, its value limited due to restricted availability in many low affluent countries (9).

The aetiology of BI can be varied: primarily a developmental bone anomaly or secondarily acquired in bone pathologies such as osteomalacia, Paget's disease or local bone destruction as seen in rheumatoid arthritis $(1,4,6)$. Additionally, BI is associated with many other neurological problems such as Chiari malformation (inferior migration of cerebellum and brain stem) and syringomyelia of the cervical spinal cord (6).

The prevalence of $\mathrm{BI}$ is known to vary with the age, ethnicity, and morphology of the posterior fossa and the 
Kodiara I, Gamage D, Ilayperuma I - Prevalence of primary basilar invagination among asymptomatic Sri Lankan adults - computed tomographic study

craniocervical junction (5). Further, the incidence of BI known to be higher in some nationalities and among different ethnic groups (2). Similar ethnic or geographical variation can be expected from Sri Lankan populations as well. Hence, the projected prevalence of BI for Sri Lankans could be either an under or overestimate of the actual values. Therefore, the knowledge on the population-specific prevalence of BI would of great value in establishing relevant guidelines to manage the asymptomatic cohort of a particular population. Thus, our study aimed to evaluate the prevalence of BI in a group of Sri Lankan adults and to assess some associated factors of BI.

\section{Material and methods}

This cross-sectional, observational study recruited all the subjects $(\mathrm{n}=915)$ presented for computed tomographic (CT) of the brain under investigation of headache, to the Radiology department of the Teaching Hospital Rathnapura, Sri Lanka, from June 2019 to November 2019. The subjects were screened using medical records and already stored CT images to identify eligibility. The exclusion criteria included; a history of epilepsy, craniofacial syndrome, cervical spinal deformity, craniocervical surgery, known craniocervical junction instability, any disease process with bone deformity (such as rheumatoid arthritis, osteomalacia, Paget's disease), head injury, cervical spine injury, brain or spinal tumours, hydrocephalus or meningoencephalitis, neuropathy, and positive neurological signs. Any subject aged less than 18 years, with incomplete CT brain study or CT image degradation for any reason or not consented subjects were also excluded from the study. Informed written consent obtained before the data collection. Patients' privacy and anonymity maintained throughout the study. The ethical clearance for the study was obtained from the Ethical Review Committee, Kothalawala Defense University, Sri Lanka.

\section{CT measurement procedure}

All the brain CT scans were performed in the same CT scanner (Toshiba Alexion, Tochigi, Japan, released to the market in 2013), evaluated by same experienced Radiologist. The measurements were obtained under adequate magnification from the sagittal reconstructed CT images of the brain in bone and soft tissue window settings, using the electronic calliper of the image processing software, to the nearest $0.1 \mathrm{~mm}$.

The Chamberlain's line was defined as the line joining the posterior edge of the hard palate and the opistium (6). To define the subjects with basilar invagination (BI) the perpendicular distance between the tip of the odontoid process and the Chamberlain's line was obtained. The BI was defined using three predetermined criteria as used in previous studies (8): BI is present if the odontoid tip is $2 \mathrm{~mm}, 5 \mathrm{~mm}$ or $6 \mathrm{~mm}$ above the Chamberlain's line (Figure 1a). The prevalence of BI was calculated under each diagnostic criterion. 
Kodiara I, Gamage D, Ilayperuma I - Prevalence of primary basilar invagination among asymptomatic Sri Lankan adults - computed tomographic study

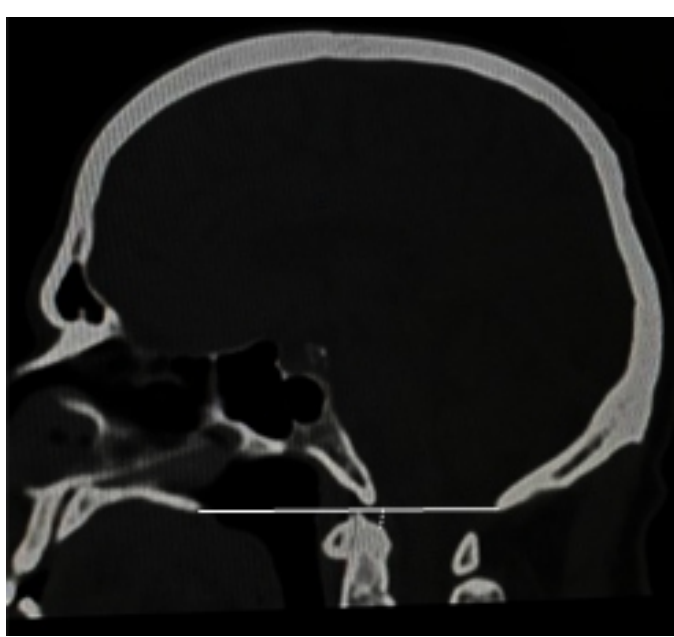

Fig 1a. Sagittal reconstructed CT image of the brain demonstrating the measurements taken to define basilar invagination. (Solid line: Chamberlain's line; dotted line: measurement obtained from the tip of the odontoid process to the Chamberlain's line).

The posterior fossa height was measured from the foramen magnum to the highest point of the tentorium, using a sagittal image of the brain in soft tissue window image settings (Figure $1 b)$.

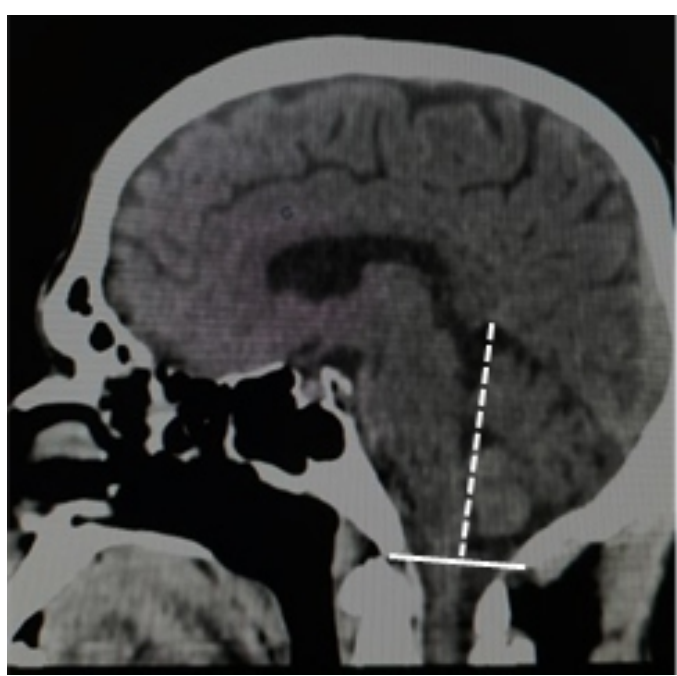

Fig 1b. Sagittal reconstructed CT image of the brain demonstrating measurement of the posterior fossa height. (Dotted line: height of the posterior fossa; solid line: line defining the position of foramen magnum).

Internal cranial height was measured from the foramen magnum to the highest point of the inner aspect of the calvarium, using a sagittal image of the brain in bone window image settings (Figure 1c). Klaus' height index was defined as the height between the tip of the odontoid process and the highest point of the tentorium (12) (Figure 1d).

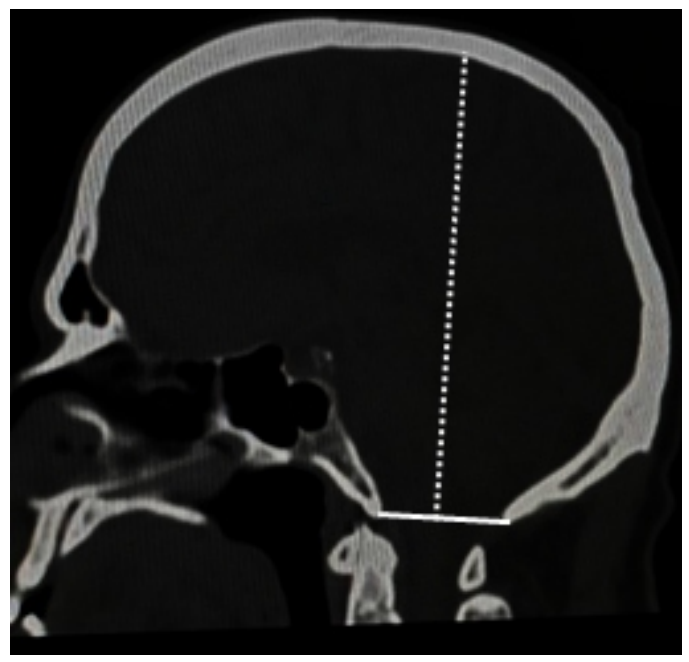

Fig 1c. Sagittal reconstructed CT image of the brain demonstrating the procedure of obtaining the measurement of internal cranial height. (Dotted line: internal cranial height measurement; solid line: the line defining the position of foramen magnum). 
Kodiara I, Gamage D, Ilayperuma I - Prevalence of primary basilar invagination among asymptomatic Sri Lankan adults - computed tomographic study

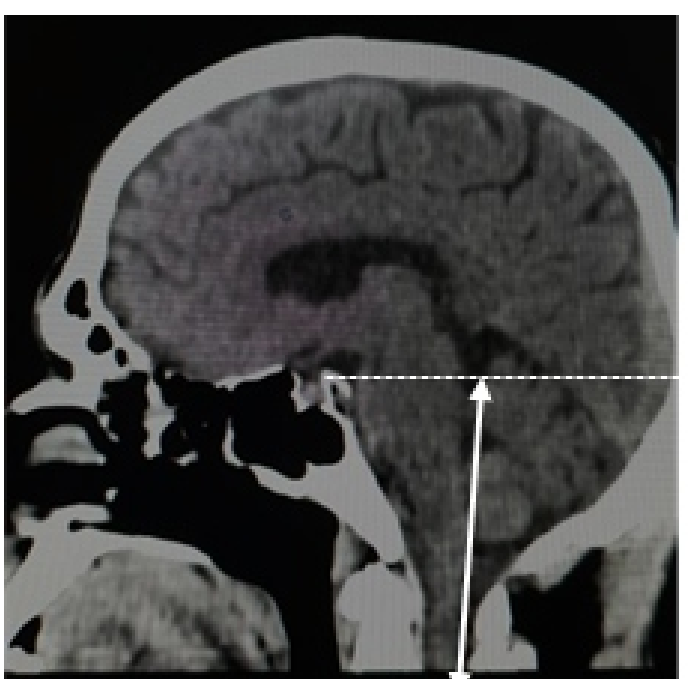

Fig 1d. The procedure of obtaining the measurement of Klaus' height index in a sagittal reconstructed CT image of the brain. (Dotted line: the line defining the highest point of the posterior fossa; arrowed line: the measurement obtained from the tip of the odontoid process to the highest position of the posterior fossa).

The position of the cerebellar tonsils to the foramen magnum delineated the criteria for diagnosing the Chiari malformation (Figure 2): the tonsillar position up to $3 \mathrm{~mm}$ below the foramen of magnum was considered normal; the tonsillar position $3-5 \mathrm{~mm}$ below the foramen of magnum was considered abnormal for symptomatic patients; the tonsillar position $5 \mathrm{~mm}$ below the foramen of magnum was considered abnormal for asymptomatic patients (6). Since our study population was asymptomatic, to define the cases of Chiari malformation, we considered the tonsillar position below $5 \mathrm{~mm}$ as abnormal.

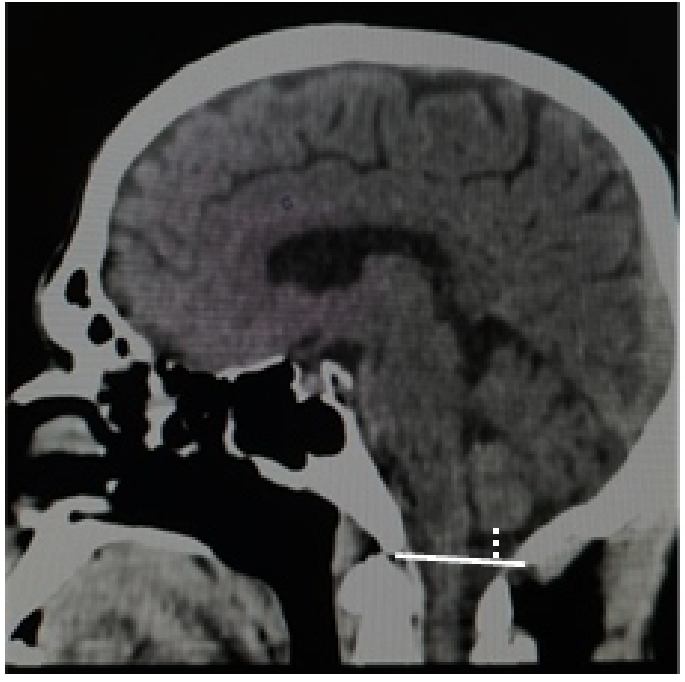

Fig 2. Sagittal reconstructed CT image of the brain demonstrating the procedure of obtaining the measurements to define the cerebellar tonsillar position. (Solid line: the line defining the position of the foramen magnum; dotted line: the measurement obtained from the foramen magnum to the lower border of the cerebellar tonsil).

\section{Statistical analysis}

Statistical analysis performed using SPSS 25 IBM statistical software. Categorical variables expressed as percentages and continuous variables as means, standard deviations and ranges. The differences between variables compared for significance using paired sample T-test and Qi-square tests, and the relationships between the variables evaluated using binary logistic regression analysis. The $\mathrm{P}$ values of less than 0.05 considered statistically significant. 
Kodiara I, Gamage D, Ilayperuma I - Prevalence of primary basilar invagination among asymptomatic Sri Lankan adults - computed tomographic study

\section{Results}

The study recruited 135 subjects, consisted of $49.6 \%(n=67)$ males and $50.4 \%(n=68)$ females, in the mean age of $56 \pm 18$ years, and the age range of 20 to 97 years. The mean age of the males was $57 \pm 17.9$ years, and the females was $55 \pm 19.0$ years, with no significant age difference between them $(\mathrm{p}=0.633)$.

The position of the tip of the odontoid process to the Chamberlain's line was evaluated. In $80.1 \%(n=109)$, the odontoid process positioned below the Chamberlain's line keeping a mean distance of $2.43 \pm 1.96 \mathrm{~mm}$ and a range of 0 to $9.7 \mathrm{~mm}$. In $19.1 \%(\mathrm{n}=26)$, the tip of the odontoid process was above Chamberlain's line; the mean distance was $2.33 \pm 1.06$ with a range of 0.6 to $4.7 \mathrm{~mm}$. The gender variation on the position of the odontoid process was assessed. The odontoid process was below the Chamberlain's line in $86.6 \%$ $(n=58)$ of males and $75 \%(n=51)$ of females demonstrating no significant $(\mathrm{X} 2=2.904 ; \mathrm{p}=0.088)$ gender variation .

The mean internal cranial height of the study population was $128.69 \pm 5.5 \mathrm{~mm}$; males' $132.03 \pm 4.8 \mathrm{~mm}$; females' $125.79 \pm 4.3 \mathrm{~mm}$. The internal cranial height of males was significantly higher than the female counterpart $(\mathrm{T}=6.81$; $\mathrm{p}<0.001)$. The mean posterior fossa height was $64.81 \pm 4.72 \mathrm{~mm}$; males' $66.36 \pm 4.4 \mathrm{~mm}$; females' $63.26 \pm 4.5 \mathrm{~mm}$. The mean Klaus' height index was $66.23 \pm 5.7 \mathrm{~mm}$; males' $68.2 \pm 5.3 \mathrm{~mm}$; females' $64.39 \pm 5.9 \mathrm{~mm}$. The posterior fossae height $(\mathrm{T}=4.016 ; \mathrm{p}<0.001)$ and Klaus height index $(\mathrm{T}=4.125 ; \mathrm{p}<0.001)$ of males were significantly higher than the female counterpart.

Table 1 depicts the distribution of basilar invagination in the study population. The incidence of basilar invagination calculated considering three cut off values: $2 \mathrm{~mm} ; 5 \mathrm{~mm}$ and 6 $\mathrm{mm}$ above the Chamberlain's line. When the tip of the odontoid process $2 \mathrm{~mm}$ above the Chamberlain's line considered abnormal $(2 \mathrm{~mm}$ as the cut off value), the incidence of basilar invagination was $11.8 \%$. However, for this population, the incidence of BI was $0 \%$, for both $5 \mathrm{~mm}$, and $6 \mathrm{~mm}$ cut of values.

(the cut of values used to identify basilar invagination by considering the position of the tip of odontoid process: $2 \mathrm{~mm}, 5 \mathrm{~mm}$ and $6 \mathrm{~mm}$ above the Chamberlain's line as abnormal (basilar invagination) in respective cases; $\mathrm{BI}$ : basilar invagination; CL: Chamberlain's line; OP: odontoid process)

Table 1: The distribution of basilar invagination in the study population for different diagnostic thresholds

\begin{tabular}{lll}
\hline & BI - present & BI - absent \\
\hline OP 2mm above CL & $11.8 \%(\mathrm{n}=16)$ & $88.2 \%(119)$ \\
OP 5mm above CL & $0 \%(\mathrm{n}=0)$ & $100 \%(135)$ \\
OP 7mm above CL & $0 \%(\mathrm{n}=0)$ & $100 \%(135)$ \\
\hline
\end{tabular}


Kodiara I, Gamage D, Ilayperuma I - Prevalence of primary basilar invagination among asymptomatic Sri Lankan adults - computed tomographic study

Table 2 describes the gender distribution of basilar invagination. There was no significant difference $(\mathrm{X} 2=1.068 ; \mathrm{p}=0.301)$ in the incidence of basilar invagination among males $(9 \%)$ and females (14.7\%). Binary logistic regression analysis performed to analyze the factors associated with the occurrence of basilar invagination (Table 3). Though the internal cranial height negatively associated with basilar invagination $(\mathrm{OR}=0.823$, $\mathrm{p}=0.044)$, none of the other evaluated factors such as age, gender, Klaus' height index or the posterior fossa height of the subject was significantly associated with the incidence (Table 2). fulfilled the diagnostic criteria of Chiari malformation.

\section{Discussion}

The value of diagnosing asymptomatic patients with basilar invagination (BI) underscore the possibility of developing neurological symptoms at any age, upon which surgical intervention often needed to prevent permanent neurological disability $(4,6,10,13)$. This pioneering study from a Sri Lankan population, reports an $11.8 \%$ incidence of primary BI among asymptomatic adults. Additionally, we found that the incidence of primary BI

Table 2: The gender distribution of basilar invagination

\begin{tabular}{lllll}
\hline & \multicolumn{2}{c}{ Male $(\mathrm{n}=67)$} & \multicolumn{2}{c}{ Female $(\mathrm{n}=68)$} \\
\hline & BI present & BI absent & BI present & BI absent \\
$2 \mathrm{~mm}$ above CL & $9 \%(\mathrm{n}=6)$ & $91 \%(\mathrm{n}=16)$ & $14.7 \%(\mathrm{n}=10)$ & $85.3 \%(\mathrm{n}=58)$ \\
$5 \mathrm{~mm}$ above CL & $100 \%(\mathrm{n}=67)$ & $0 \%(\mathrm{n}=0)$ & $0 \%(\mathrm{n}=0)$ & $100 \%(\mathrm{n}=68)$ \\
$6 \mathrm{~mm}$ above CL & $100 \%(\mathrm{n}=67)$ & $0 \%(\mathrm{n}=0)$ & $0 \%(\mathrm{n}=0)$ & $100 \%(\mathrm{n}=68)$ \\
\hline
\end{tabular}

(the cut of values used to identify basilar invagination by considering the tip of the odontoid process $2 \mathrm{~mm}, 5 \mathrm{~mm}$ and $6 \mathrm{~mm}$ above the Chamberlain line.

The mean position of the cerebellar tonsils was $4.19 \pm 2.42 \mathrm{~mm}$ above the foramen magnum. The tonsilar position ranged from $14 \mathrm{~mm}$ above to $3 \mathrm{~mm}$ below the foramen of magnum. However, the majority $(97.8 \% ; n=131)$ had cerebellar tonsils placed above the foramen of magnum. Despite having three patients $(2.2 \% ; n=3)$ who were with the cerebellar tonsils placed below the foramen of magnum, none $(0 \%)$ has a negative association with the internal cranial height. The clinical prediction of asymptomatic BI renders difficult due to lack of influence on age, gender, posterior fossa height or Klaus' height index.

The general examination findings of patients with BI are non-specific, would present with a short or deformed neck (for example, torticollis) that share with many other congenital and acquired neck disorders (14). The majority of BI patients are asymptomatic when they are symptomatic often present with non-specific symptoms while hiding the 
Kodiara I, Gamage D, Ilayperuma I - Prevalence of primary basilar invagination among asymptomatic Sri Lankan adults - computed tomographic study

real potential of progressing into neurological disabilities. Therefore, a high degree of suspicion is essential in case detection. Knowing the prevalence of BI in the general population would facilitate case detection and disability prevention that may be triggered upon a minor trauma $(2,5,8,9)$. Further to this, the reported ethnic variations observed in prevalence devalue the use of references from other populations (2) and highlight the need to have population-specific prevalence values of asymptomatic BI.

Proving the hypothesis, we found an ethnic variation in the prevalence of BI by reporting $(11.8 \%$ prevalence of asymptomatic $\mathrm{BI}$ ) lower values than Brazilians (37.5\%, $n=40)(8)$. The Brazilian study has employed three different protocols to define the cases of BI (8). The standard protocol uses the tip of the odontoid process $2 \mathrm{~mm}$ above the Chamberlain's line as abnormal $(5,8)$. In the other two protocols, the odontoid tip placed $5 \mathrm{~mm}$ and $6 \mathrm{~mm}$ above the Chamberlain's line were considered abnormal (8). Our population showed different prevalence values in all criteria: while asymptomatic Brazilians were having $10 \%$ (5mm above the Chamberlain's line) and 5\% (6mm above the Chamberlain's line), we found none fulfilling the same criteria in our sample. Data regarding the true incidence of BI among the general population scares, some stated it as low as $1 \%$ (13). Importantly, data on the incidence in the Indian subcontinent is yet to discover. Hence, the findings of this study would be considered as the references for the Indian subcontinent.
Among many associations of BI, small posterior fossa volume, platybasia (abnormal angle between the anterior skull base and the clivus), clival hypoplasia, condylar hypoplasia, hypoplastic Atlas, Chiari malformation and syringomyelia were frequent $(2,4-$ $6,14,15)$. However, we found that the indicators of posterior fossa volume, such as Klaus' height index, and posterior fossa height were not associated with BI of this cohort. Interestingly, it was observed that the reduced internal cranial height have a positive association with BI. This fact may also represent ethnic influence on anthropometry.

A positive association has been established among BI, reduced posterior fossa height and Chiari malformation (6). Though a high incidence ( $90 \%$ ) of Chiari malformation reported among BI patients, none of our patients $(0 \%)$ diagnosed to have Chiari malformation $(6,16,17)$. The observed discrepancy in the incidence of Chiari malformation may partly represent the differences in methodology; this study assessed asymptomatic individuals, while the other studied symptomatic patients. The neurological symptoms classically associated with the compression of vital structures in the craniocervical region (such as cerebellar tonsils, brainstem and or the cervical spinal cord); the possibility of compression probably aggravated by the low posterior fossa volume $(6,15)$. The present study cohort being asymptomatic possibly explains the lower incidence of Chiari malformation. 
Kodiara I, Gamage D, Ilayperuma I - Prevalence of primary basilar invagination among asymptomatic Sri Lankan adults - computed tomographic study

Since the clinical prediction of $\mathrm{BI}$ is difficult, the mainstay of diagnosis is with imaging, for that different modality contribute uniquely (6). The plain radiograph is less sensitive in delineating bone landmarks such as the posterior edge of the hard palate and the opistium than cross-sectional imaging -the measurements from the Chamberlain's line more accurately taken with CT images $(9,10)$. The associated soft tissue abnormalities such as Chiari malformation and syringomyelia are better evaluated with MRI than with CT. Improved imaging technologies such as CT and MRI enhance the yield of assessing associated bone abnormalities and neural compression $(1,4)$. Additionally, dynamic evaluation of the craniocervical junction enables diagnosing functional craniocervical junction instability; though there are different degrees of sensitivity, almost all the imaging modalities allow the dynamic assessment $(1,4,8,9,14)$. Craniocervical junction instability said to be the critical most factor in developing many symptoms and disabilities of BI $(2,4,6,8,9)$. Symptomatic frequency of BI is high in severe degrees of superior odontoid migrations, particularly when associated with atlantoaxial facet dislocation (5). Both abnormalities - the degree of odontoid migration and atlantoaxial facet dislocations - are reliably evaluated using cross-sectional imaging modalities such as $\mathrm{CT}$ and MRI.

The surgical intervention being the cornerstone of management in $\mathrm{BI}$ is generally offered for symptomatic patients, particularly those who are with an unstable craniocervical junction. Pain, restricted movements and hyper-lordosis of the neck are recognized features of the unstable craniocervical junction (15). Rather than restoring the anatomy, surgery primarily aimed to realign and stabilize the craniocervical junction by fixing the atlantoaxial junction (14). A screening imaging procedure would be of value to identify craniocervical junction instability among asymptomatic patients and those who are with nonspecific external appearances such as short or deformed necks. The lateral view of the X-ray cervical spine obtained in neck flexion and extension is a readily available, cheapest imaging modality, which has the ability to indicates the craniocervical junction instability reliably. However, CT and MRI provide a dynamic assessment with a much higher sensitivity (14).

The reliability of the findings of the current study is confirmed because the diagnostic accuracy of BI with sagittal reconstructed $\mathrm{CT}$ of the craniocervical junction is high (5). Despite, this being a pioneer study to document the prevalence of basilar invagination for a Sri Lankan population, it suffers from several limitations. Firstly, being a single centre study limits generalizing the findings to the Sri Lankan population, which could be better achieved in the multi-centric study. Secondly, though it is vital to know the prevalence of $\mathrm{BI}$ in asymptomatic individuals, clinical implications would be enhanced if combined with 
Kodiara I, Gamage D, Ilayperuma I - Prevalence of primary basilar invagination among asymptomatic Sri Lankan adults - computed tomographic study

functional assessment, such as assessment of the atlantoaxial instability.

In conclusion, the high asymptomatic prevalence $(11.8 \%)$ of basilar invagination reflects the need for having a high degree of suspicion to detect patients with non-specific symptoms.

\section{Conflict of interest}

None declared.

\section{Acknowledgments}

The authors wish to acknowledge Faculty of Medicine, University of Ruhuna Research Grant-2019 for financial assistance.

\section{Correspondence}

Dr. Iroshani Kodikara,

Senior Lecturer in Anatomy \&

Consultant Radiologist,

Department of Anatomy,

Faculty of Medicine,

University of Ruhuna,

Galle, Sri Lanka.

E-mail: iroshani.kodikara@gmail.com

Tel: +94 718390027

\section{References}

1. Smoker WR. Craniovertebral junction: normal anatomy, craniometry, and congenital anomalies. Radiographics. 1994;14(2):255-277.

2. Goel A. Facetal alignment: Basis of an alternative Goel's classification of basilar invaginationJ Craniovertebr Junction Spine. 2014 Apr-Jun; 5(2): 59-64.
3. Lopez AJ, Scheer JK, Leibl KE, Smith ZA, Dlouhy BJ, Dahdaleh NS. Anatomy and biomechanics of the craniovertebral junction. Neurosurg Focus. 2015;38(4):1-8.

4. Wang YQ, FAN T, Zhao XG, Liang C, Cai QF, Zhao HJ. Surgical treatment of congenital basilar invagination with different pathological characteristics: Report of 139 cases. Transl. Neurosci.

Clin. 2017, 3(4): 204-212.

5. Goel A. Basilar invagination, Chiari malformation, syringomyelia: A review. Neurol India. 2009;57:235-246.

6. De Oliveira Brito JNP, Dos Santos BA, Nascimento IF, Martins LA, Tavares CB. Basilar invagination associated with chiari malformation type I: A literature review. Clinics. 2019;74:1-6.

7. Pearce JMS. Platybasia and basilar invagination. Eur Neurol. 2007;58(1):62-64.

8. Joaquim AF. Management of Basilar Invagination. Jbnc - J Bras Neurocir. 2018;24(1):53-59.

9. Joaquim AF, Fernandes YB, Mathias RN, et al. Incidência da invaginação basilar com heriação tonsilar - Estudo da craniometria do tipo caso-controle. Arq Neuropsiquiatr. 2014;72(9):706711.

10. Guo X, Han Z, Xiao J, et al. Cervicomedullary angle as an independent radiological predictor 
Kodiara I, Gamage D, Ilayperuma I - Prevalence of primary basilar invagination among asymptomatic Sri Lankan adults - computed tomographic study

of postoperative neurological outcome in type A basilar invagination. Scientific reports. 2019:1-7.

11. Samartzis D, Kalluri P, Herman J, Lubicky JP, Shen FH. Superior odontoid migration in the KlippelFeil patient. Eur Spine J. 2007;16(9):1489-1497.

12. Bell D, Vankatesh M. Kaus height index. Radiopedia web site reviewed on 24.03.2020.

13. Mourad F, Giovannico G, Maselli F, Bonetti F, Fernández De Las Peñas C, Dunning J. Basilar impression presenting as intermittent mechanical neck pain: A rare case report Rehabilitation, physical therapy and occupational health. BMC Musculoskelet Disord. 2016;17(1):4-8.

14. Shah A, Serchi E. Management of basilar invagination: A historical perspective. J Craniovertebr

Junction Spine. 2016; 7(2): 96-100.

15. Milhorat TH, Chou MW, Trinidad EM, Kula RW, Mandell M, Wolpert C, et al. Chiari I malformation redefined: clinical and radiographic findings for 364 symptomatic patients.

Neurosurgery. 1999;44(5):1005-17

16. Elster AD, Chen MY. Chiari I malformations: clinical and radiologic reappraisal. Radiology. 1992;183 (2):347-53.

17. da Silva JA, dos Santos AA Jr, Melo LR, de Araújo AF, Regueira GP. Posterior fossa decompression with tonsillectomy in 104 cases of basilar impression, Chiari malformation and/or syringomyelia. Arq Neuropsiquiatr. 2011;69 (5):817-23. 